\title{
Multicenter Prevalence Study Comparing Molecular and Toxin Assays for Clostridioides difficile Surveillance, Switzerland
}

\author{
Andreas F. Widmer, Reno Frei, Ed J. Kuijper, Mark H. Wilcox, Ruth Schindler, \\ Violeta Spaniol, Daniel Goldenberger, Adrian Egli, Sarah Tschudin-Sutter
}

\begin{abstract}
Public health authorities in the United States and Europe recommend surveillance for Clostridioides difficile infections among hospitalized patients, but differing diagnostic algorithms can hamper comparisons between institutions and countries. We compared surveillance based on detection of $C$. difficile by PCR or enzyme immunoassay (EIA) in a nationwide $C$. difficile prevalence study in Switzerland. We included all routinely collected stool samples from hospitalized patients with diarrhea in 76 hospitals in Switzerland on 2 days, 1 in winter and 1 in summer, in 2015. EIA C. difficile detection rates were 6.4 cases $/ 10,000$ patient bed-days in winter and 5.7 cases/10,000 patient bed-days in summer. PCR detection rates were 11.4 cases $/ 10,000$ patient bed-days in winter and 7.1 cases $/ 10,000$ patient bed-days in summer. We found PCR used alone increased reported $C$. difficile prevalence rates by $\leq 80 \%$ compared with a 2 -stage EIAbased algorithm.
\end{abstract}

Since its identification as a cause of antibiotic-asSociated pseudomembraneous colitis in 1978 (1), Clostridioides difficile has emerged as a major healthcare-associated pathogen worldwide. In the United States, C. difficile infection (CDI) rates doubled during 1996-2003 (2), and rates of CDI were reported to be 76.9 cases $/ 10,000$ discharges in 2005 (3). In a more recent national point-prevalence study including US healthcare facility in-patients, 13/1,000 patients were found to be either infected or colonized (4), a higher rate than had been previously estimated. In a national

Author affiliations: University Hospital Basel, Basel, Switzerland (A.F. Widmer, R. Frei, R. Schindler, V. Spaniol, D. Goldenberger,

A. Egli, S. Tschudin-Sutter); Leiden University Medical Center, Leiden, the Netherlands (E.J. Kuijper); Leeds Institute of Biomedical and Clinical Sciences, University of Leeds, and Leeds Teaching Hospitals, Leeds, UK (M.H. Wilcox)

DOI: https://doi.org/10.3201/eid2610.190804 point-prevalence study of nosocomial infections in the United States, $C$. difficile was the most common causative pathogen overall (5). The increase largely has been attributed to the emergence of the hypervirulent strain, PCR ribotype 027 (RT027), which was identified as causative strain in $82 \%$ of cases during CDI outbreaks in Quebec, Canada, during 2001-2003 and accounted for $31 \%$ of all cases of healthcare-associated infections in the United States in 2011 (6-9). In Europe, CDI incidence varies across hospitals and countries with a weighted mean of 4.1 cases $/ 10,000$ patient-days per hospital in 2008 (10). The most recent study on CDI prevalence in Europe suggests an increase in the number of cases, reporting a mean of 7.0 cases $/ 10,000$ patient-bed days and ranging among countries from 0.7 to 28.7 cases $/ 10,000$ patient-bed days (11). The most common ribotype identified was RT027, which was detected in 4 countries: Germany, Hungary, Poland, and Romania (11).

To estimate and compare the burden of CDI across the United States, the US Centers for Disease Control and Prevention (CDC) began populationbased CDI surveillance in 10 locations in 2011 (12). The European Centre for Disease Prevention and Control (ECDC) began coordinating CDI surveillance in acute care hospitals in Europe in 2016 (13). Both authorities provide case definitions based on different diagnostic approaches, including detection of $C$. difficile toxin $A$ and $B$ by enzyme immunoassay (EIA) or detection of toxin-producing $C$. difficile organisms by PCR. However, the use of different diagnostic algorithms to detect $C$. difficile might hamper comparisons between institutions and countries. Therefore, in a nationwide $C$. difficile multicenter prevalence study in Switzerland, we systematically compared surveillance measures based on detection of $C$. difficile in stool by either PCR as a stand-alone 
test or by a 2-stage algorithm consisting of an EIA to detect glutamate dehydrogenase $(\mathrm{GDH})$ and toxins $\mathrm{A}$ and $\mathrm{B}$.

\section{Methods}

\section{Study Design}

We performed a nationwide multicenter prevalence study of toxigenic $C$. difficile detected in stool samples routinely collected from hospitalized patients with diarrhea. Our study followed the design of a previous point-prevalence study for maximal comparibility between our results and data from Europe (11). University Hospital Basel, a tertiary care center in Switzerland, coordinated the study. All hospitals participating in Swissnoso (https://www.swissnoso.ch), a national infection prevention network, were asked to participate. The Swissnoso network consists of 85 acute care hospitals that account for a total of 26,341 beds.
The Ethics Committee Northwest and Central Switzerland (Ethikkommission Nordwest-und Zentralschweiz) issued a declaration of no objection for this study. We adhered to STROBE guidelines for reporting on observational studies (14).

\section{Sample Collection}

All stool samples collected from inpatients $>1$ year of age with diarrhea that were submitted to the microbiology laboratory on 2 specified sampling days, 1 in winter and 1 in summer, in 2015 were eligble for inclusion. Only 1 sample per patient was included. In addition, stool samples that tested positive for toxigenic $C$. difficile $\leq 1$ week prior to each study day also were collected from all institutions to obtain a more detailed estimate of ribotype distribution in Switzerland.

We collected the following institutional data for all hospitals and their affiliated microbiology laboratories: contact information; detailed information

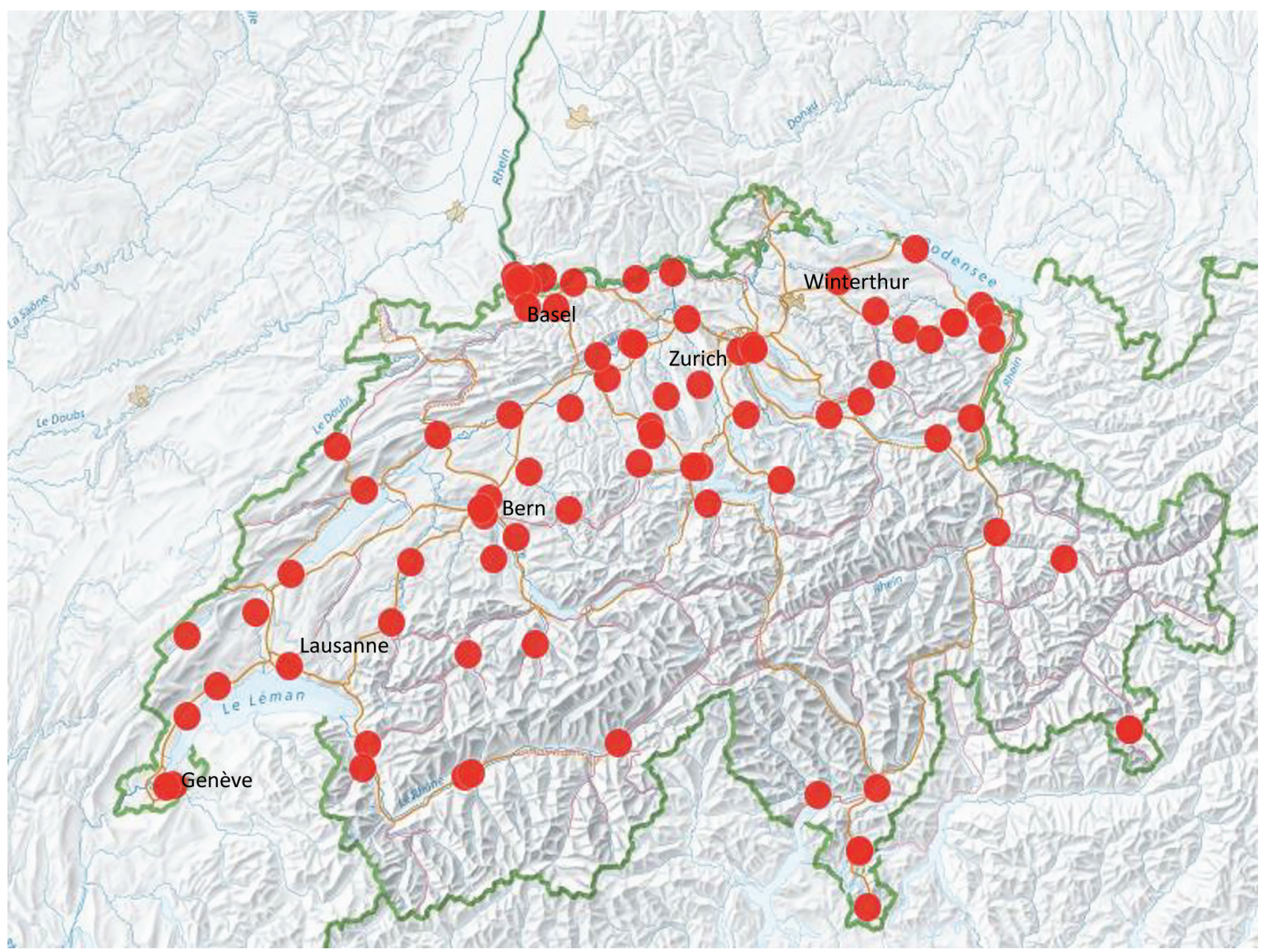

Figure 1. Distribution of centers participating in a prevalence study comparing molecular and toxin assays for nationwide surveillance of Clostridioides difficile, Switzerland. Red circles represent location of participating centers. 
regarding laboratory diagnostics in place; and detailed information on the total number of admissions, number of beds, and number of patients hospitalized on the 2 days of the study. We also collected information on the total number of diagnosed CDI cases at each institution during the study year. For each eligible stool sample, we collected the following data: date of sample collection, age and gender of patient, ward location and clinical specialty, institution, whether a $C$. difficile test was ordered by the treating physician, and result of the $C$. difficile test if testing was performed at the local laboratory.

\section{Procedures}

We tested all stool samples at the Division of Clinical Microbiology of the University Hospital Basel by using a 2-stage algorithm consisting of EIA and PCR. We performed EIA to detect GDH and toxins A and B by using C. DIFF QUIK CHEK COMPLETE (Techlab, https://www.techlab.com), following the manufacturer's instructions. We then performed PCR to detect the toxin B gene by using the RealStar PCR Kit (Altona Diagnostics, https://www.altona-diagnostics. com). For detected $C$. difficile, we performed strain typing by using high-resolution capillary gel-based PCR ribotyping according to the method previously described by Stubbs et al. (15).

\section{Outcomes}

We calculated reported and measured rates of detected toxigenic C. difficile per 10,000 patient bed-days across participating institutions. We compared differences in testing algorithms for detection of toxigenic $C$. difficile across institutions in Switzerland and performance characteristics of diagnostic algorithms. We considered the proportion of missed toxigenic $C$. difficile cases and ribotype distributions as additional outcomes. We further assessed the proportion of laboratories using optimized C. difficile diagnostic tests, which we defined as using an algorithm recommended by the European Society of Clinical Microbiology and Infectious Diseases (16).

\section{Statistical Analyses}

We separately calculated rates for each diagnostic algorithm performed in the coordinating center laboratory. In addition, we separately calculated rates for dedicated children's hospitals. We defined missed $C$. difficile cases as those in which tests were negative at the participating hospital's laboratory but positive at our institution. We used descriptive statistics to report ribotypes and differences in diagnostic

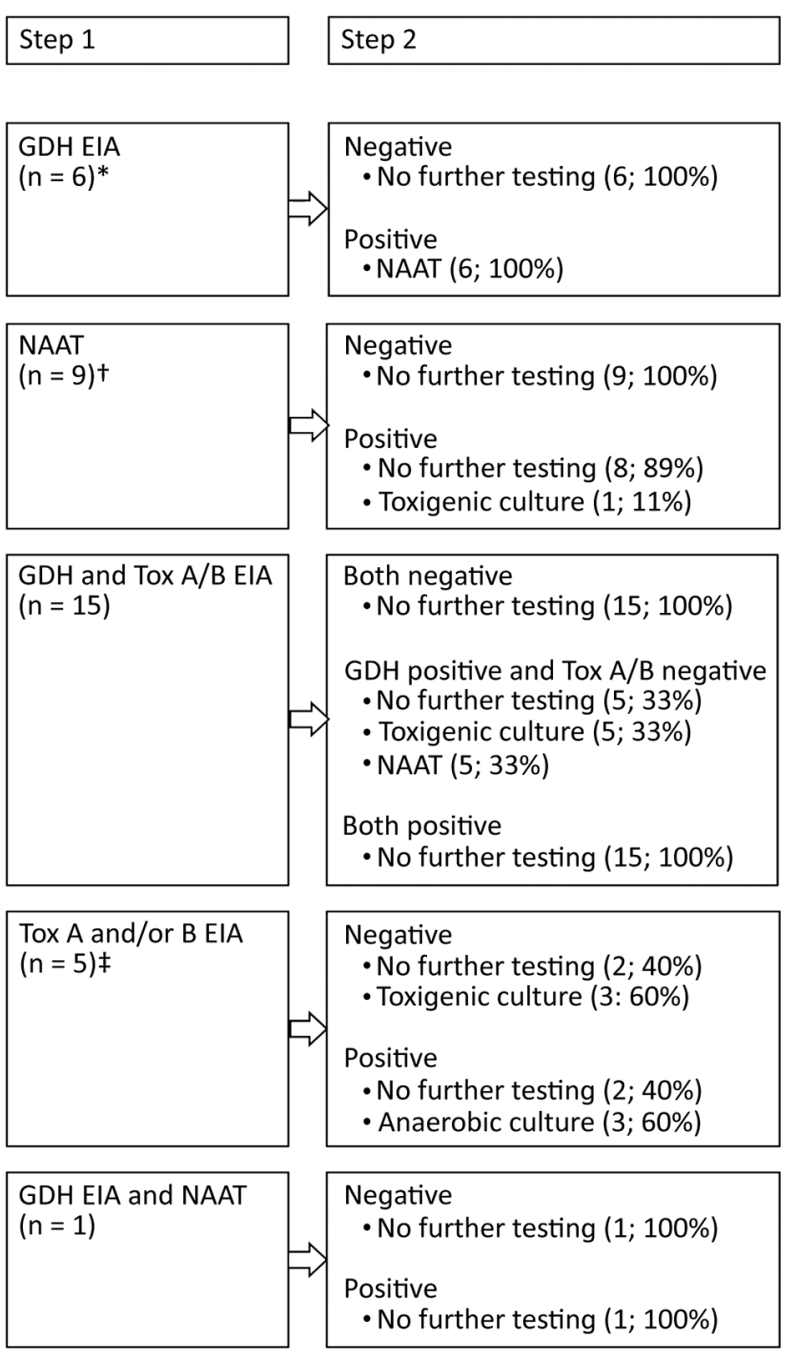

Figure 2. Testing algorithms at the 36 laboratories participating in a prevalence study comparing molecular and toxin assays for nationwide surveillance of Clostridioides difficile, Switzerland. EIA, enzyme immunoassay; GDH, glutamate dehydrogenase; NAAT, nucleic acid amplification test; Tox, toxin. *Seven samples taken during the summer sampling period. †Ten samples taken during the summer sampling period. $\ddagger$ Three samples taken during the summer sampling period.

algorithms across all participating institutions. All analyses were perfomed in Stata version 15.1 (StataCorp,https:/ / www.stata.com).

\section{Results}

Participating institutions included 76/85 (89.4\%) institutions belonging to the Swissnoso network. Among participating institutions, 5 were academic teaching hospitals, 3 were dedicated children's hospitals, and 36 were affiliated microbiology laboratories. Participating institutions were distributed across all geographic regions of Switzerland (Figure 1). 
Participating institutions reported collecting a fecal sample for microbiological workup in $\approx 65 \%$ (SD $\pm 25 \%)$ of all patients with hospital-onset diarrhea. Among participating institutions, 15/76 (19.7\%) did not begin CDI treatment before fecal sample collection. Among institutions that intitiated treatment before collecting fecal samples, 23/76 (30.3\%) began treatment in $<2 \%$ of patients, $12 / 76(15.8 \%)$ began treatment in $3 \%-5 \%$ of patients, $8 / 76(10.5 \%)$ began treatment in $6 \%-10 \%$ of patients, and $1(1.3 \%)$ began treatment in $11 \%-20 \%$ of patients. The other $17(22 \%)$ institutions were not able to provide an estimate of these data.

Overall, 354 stool samples were submitted to the coordinating center, of which 338 were eligible for study inclusion; 16 samples were excluded because they were not liquid, their submitted data were incomplete, or they were duplicate samples from 1 patient. Among 338 samples included, 250 were collected as part of the point-prevalence study. We excluded 8 of these because the samples were collected from patients $<1$ year of age. In all, we included 242 samples in the point-prevalence study.

\section{Diagnostic Algorithms}

Among the 36 participating laboratories, 1 routinely tested all diarrheal stool samples for toxigenic $C$. difficile and 35 tested only if a specific test was requested. Optimized diagnostic tests for detection of toxigenic C. difficile were used by $58 \%(21 / 36)$ of laboratories in the winter sampling period and by $61 \%(22 / 36)$ in the summer sampling period. Among laboratories not following the recommendations of the European Society of Clinical Microbiology and Infectious Diseases (16), 9 in the winter sampling period and 10 in the summer sampling period used a nucleic acid amplification test (NAAT) alone, and 5 in the winter sampling period and 3 in the summer sampling period used EIAs for A and B toxins either as a standalone test or as an initial screening test. Only 1 laboratory reported having established PCR ribotyping methodologies (Figure 2).

\section{Point-Prevalence Analyses}

We collected demographic characteristics of patients whose stool samples tested positive by our testing algorithms (Table 1). C. difficile tests were required and performed for $68 \%(165 / 242)$ of stool samples; $6 \%$ $(27 / 165)$ were reported as positive by the affiliated microbiology laboratory.

At the coordinating center, we detected C. difficile in $9 \%(21 / 242)$ of samples by EIA for GDH and A and B toxins and in 12\% (30/242) of samples by PCR alone. Among all 27 samples reported as positive by the participating centers, we confirmed 18 (67\%) by EIA and $24(89 \%)$ by PCR. Among 138 samples reported as negative by the participating centers, 1 (1\%) sample tested positive by EIA and $3(2 \%)$ tested positive by PCR at the coordinating center. Among 77 samples not tested for $C$. difficile at the participating centers, we detected $C$. difficile in $2(3 \%)$ by EIA and in $3(4 \%)$ by PCR. Among 21 stool samples that tested positive by EIA at the coordinating center, a $\mathrm{C}$. difficile test was not requested in $2(10 \%)$ cases. Among 30 samples that tested positive by PCR at the coordinating center, a $C$. difficile test was not requested in 3 cases $(10 \%$; Table 2$)$.

Measured detection and testing rates of toxigenic C. difficile were higher than the reported rates across all participating institutions (Table 3). Depending on the diagnostic algorithm applied, the largest difference in prevalence across all institutions was measured during the winter sampling period, which had a prevalence of 6.4 cases $/ 10,000$ patient bed-days by EIA and 11.4 cases/10,000 patient bed-days by PCR alone. Thus, across all institutions, rates of toxigenic

\begin{tabular}{|c|c|c|c|}
\hline \multirow[b]{2}{*}{ Demographics } & \multirow[b]{2}{*}{ All patients } & \multicolumn{2}{|c|}{ Method of Clostridioides difficile detection } \\
\hline & & EIA for GDH and $A$ and $B$ toxins, $n=21$ & $\mathrm{PCR}, \mathrm{n}=30$ \\
\hline Median age, y (IQR) & $63(44-80)$ & $79(59-86)$ & $78(55-85)$ \\
\hline \multicolumn{4}{|l|}{ Sex } \\
\hline M & $104(43.0)$ & $6(28.6)$ & $10(33.3)$ \\
\hline $\mathrm{F}$ & $131(54.1)$ & $15(71.4)$ & $20(66.7)$ \\
\hline Not reported & $7(2.9)$ & 0 & 0 \\
\hline \multicolumn{4}{|l|}{ Clinical specialty } \\
\hline Medical & $127(52.5)$ & $11(52.4)$ & $11(36.7)$ \\
\hline Surgery & $43(17.8)$ & $3(14.3)$ & $6(20.0)$ \\
\hline Obstetrics, gynocology & $3(1.2)$ & 0 & 0 \\
\hline Pediatrics & $21(8.7)$ & $1(4.8)$ & $3(10.0)$ \\
\hline Other & $28(11.6)$ & $5(23.8)$ & $7(23.3)$ \\
\hline Not reported & $20(8.3)$ & $1(4.8)$ & $3(10.0)$ \\
\hline Intensive care & $40(16.5)$ & $5(23.8)$ & $5(16.7)$ \\
\hline
\end{tabular}

\footnotetext{
*Values are reported as no. (\%) except where indicated. EIA, enzyme immunoassay; GDH, glutamate dehydrogenase; IQR, Interquartile range.
} 
Table 2. Underdiagnosis and misdiagnosis of Clostridioides difficile infection at participating hospitals, Switzerland*

\begin{tabular}{lccccc}
\hline Method of detection & $\begin{array}{c}\text { No. samples } \\
\text { submitted }\end{array}$ & $\begin{array}{c}\text { No. samples } \\
\text { tested }\end{array}$ & $\begin{array}{c}\text { Undiagnosed, no. (\% of } \\
\text { all positive samples) }\end{array}$ & $\begin{array}{c}\text { False-positive, } \\
\text { no. (\%) }\end{array}$ & $\begin{array}{c}\text { False-negative, } \\
\text { no. (\%) }\end{array}$ \\
\hline EIA for GDH and A and B toxins & 242 & 165 & $2(9.5)$ & $9(5.5)$ & $1(0.6)$ \\
PCR & 242 & 165 & $3(10)$ & $3(1.8)$ & $3(1.8)$ \\
\hline${ }^{*}$ EIA, enzyme immunoassay; GDH, glutamate dehydrogenase. & & & & \\
\hline
\end{tabular}

C. difficile detection by PCR alone were $\leq 80 \%$ higher than detection rates by EIA for GDH and A and $\mathrm{B}$ toxins. In addition, detection rates by PCR alone were $\leq 100 \%$ higher in dedicated children's hospitals (Table 3).

\section{Ribotype Distribution}

We cultured and ribotyped 107 toxigenic C. difficile strains, 29 from the 2 point-prevalence days and 78 collected $\leq 1$ week before each prevalence day. We identified a large diversity of $C$. difficile ribotypes, 23 (22\%) had not been referenced before. The ribotypes most commonly identified included RT014 (12/107; $11 \%)$, presumably hypervirulent RT078 (9/107; 8\%), RT001 (7/107; 7\%), and RT002 (7/107; 7\%) (Figure 3).

\section{Discussion}

In this nationwide multicenter study, we found that PCR as a stand-alone test increased reported C. difficile prevalence rates $\leq 80 \%$ compared with a 2 -stage EIA-based algorithm. At first glance, this finding was not surprising given the higher sensitivity of EIA (16). However, the fact that our results and conclusions are based on a nationwide cohort representing all geographic regions of Switzerland adds to the study's credibility. In addition, our results strengthen the advice of the European Society of Clinical Microbiology and Infectious Diseases study group for $C$. difficile against use of a single commercial test for diagnosing CDI because of the low positive predictive values when CDI prevalence is low, $46 \%$ at a CDI prevalence of $5 \%$ (16). However, CDC and ECDC protocols for CDI surveillance define a case of CDI as the combination of diarrheal stool and a positive PCR $(12,13)$. In addition, the clinical practice guidelines for CDI in adults and children published by the Infectious Diseases Society of America and Society for Healthcare Epidemiology of America recommend testing by different approaches, such as multistep algorithms or NAAT, depending on the degree of clinical suspicion (17). Based on a systematic review and meta-analysis, the American Society of Microbiology also recommends different approaches, including NAAT-only testing, and algorithms that include GDH and NAAT or GDH, toxins, and NAAT (18). Although these recommendations stand to reason for detection of CDI in individual patients, our results challenge their utility for meaningful comparisons in surveillance studies and suggest that uniform definitions should be provided.

On both point-prevalence days, we noted a higher nationwide rate of toxigenic $C$. difficile than previously reported in incidence studies performed at different institutions in Switzerland (19-21). Our findings suggest that CDI rates have increased during the last decade in Switzerland, a finding that is in line with reports from other countries in Europe (11). Using the same diagnostic algorithm, diagnostic test, and a similar study design to the multicenter point-prevalence study of CDI in hospitalized patients with diarrhea in Europe, the nationwide mean prevalence rates are comparable in Switzerland (mean 6.1 cases/10,000 patient bed-days) and Europe (7.0 cases/10,000 patient bed-days) (11). Because we only included liquid stools in our study, our mean prevalence rate of 9.3 cases $/ 10,000$ patient bed-days measured by PCR fulfills the ECDC case definition and further shows that CDI is increasing substantially nationwide.

We found a lower proportion of missed detection of toxigenic C. difficile in Switzerland (9.5\%), driven by the absence of clinical suspicion, compared with Europe (23\%), which equates to 1 missed case of $C$. difficile per day among the included institutions in Switzerland. False-negative testing accounted for 1

\begin{tabular}{|c|c|c|c|c|c|}
\hline $\begin{array}{l}\text { Institutions and testing } \\
\text { methods }\end{array}$ & $\begin{array}{c}\text { Reported } \\
\text { rate/10,000 } \\
\text { patient bed-days }\end{array}$ & $\begin{array}{l}\text { Measured rate/10,000 } \\
\text { patient bed-days, } \\
\text { winter (range) }\end{array}$ & $\begin{array}{l}\text { Measured rate/10,000 } \\
\text { patient bed-days, } \\
\text { summer (range) }\end{array}$ & $\begin{array}{l}\text { Mean measured } \\
\text { rate/10,000 patient } \\
\text { bed-days (range) }\end{array}$ & $\begin{array}{c}\text { Testing } \\
\text { rate/10,000 patient } \\
\text { bed-days (range) }\end{array}$ \\
\hline All institutions & $3.8(0-11)$ & & & & $67.5(0-3,202)$ \\
\hline EIA & & $6.4(0-387)$ & $5.7(0-475)$ & $6.1(0-475)$ & \\
\hline NAAT & & $11.4(0-387)$ & $7.1(0-475)$ & $9.3(0-475)$ & \\
\hline Children's hospitals & $1.1(0.4-1.1)$ & & & & $22.5(7.0-46.7)$ \\
\hline EIA & & $33.7(0-73)$ & 0 & $16.9(0-73)$ & \\
\hline NAAT & & $67.3(0-99)$ & 0 & $33.6(0-99)$ & \\
\hline
\end{tabular}

EIA, enzyme immuno assay; GDH, glutamate dehydrogenase; NAAT, nucleic acid amplification tests. 


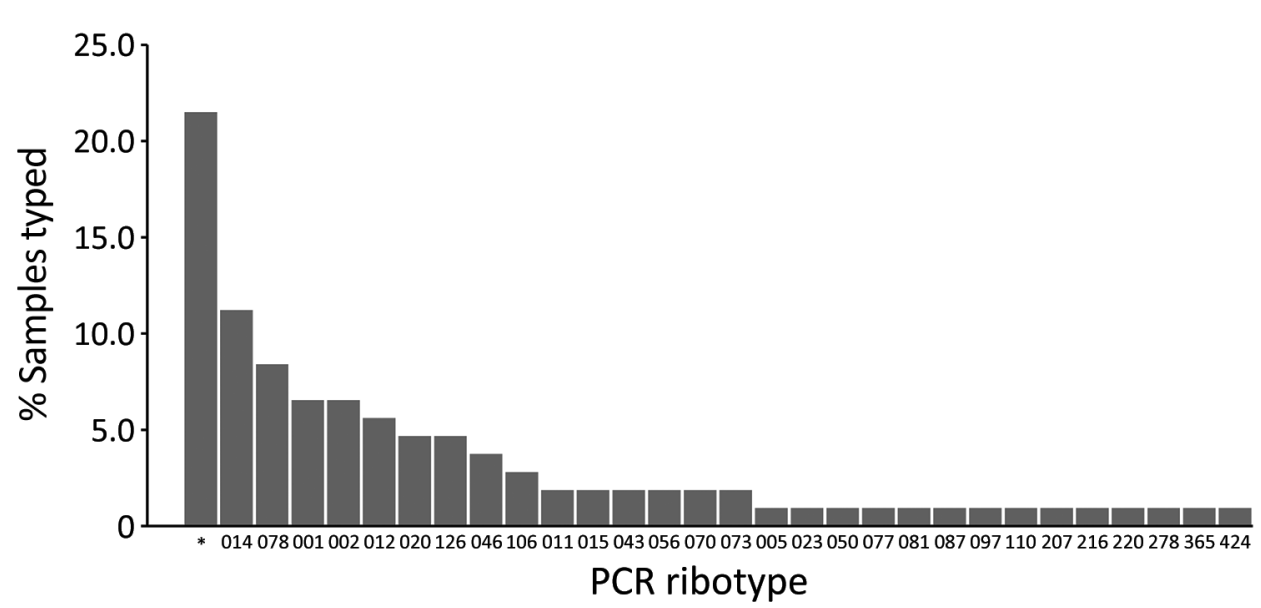

Figure 3. Distribution of PCR ribotypes among 107 samples collected in a prevalence study comparing molecular and toxin assays for nationwide surveillance of Clostridioides difficile, Switzerland. *Unknown ribotype.

additional missed diagnosis during both point-prevalence days, which extrapolates to $\approx 550$ missed cases of C. difficile per year among hospitals across the nation.

We detected a variety of different RTs during our study period, $21 \%$ of which had not been referenced before. Of note, we did not recover hypervirulent RT027, but RT078 was the third most common strain circulating in Switzerland during our study. In contrast, a point-prevalence study in Europe identified RT027 as the most commonly circulating strain during its study period but did not detect RT078. RT078 has been associated with similarly severe disease manifestations as RT027, but RT078 has been reported to affect younger patients and to be linked more commonly with community-associated disease in the Netherlands (22). RT078 has been isolated from piglets with diarrhea, possibly suggesting ongoing transmission by introduction to the food chain because isolates from humans and pigs were found to be highly genetically related (22). A component of RT078 infections also was reported in Northern Ireland, which has a large pig population and $\approx 1: 1$ ratio of cattle to humans (23). In Switzerland, RT078 has been isolated previously from 6 wastewater treatment plants, suggesting its dissemination in the community (24). Except for both hypervirulent RT027 and RT078, we identified other similarities in RT distribution between Switzerland and the rest of Europe; RT014, RT001, RT002, and RT020 were among the 10 most commonly identified ribotypes in both settings.

Our study has some limitations, most of which are intrinsic to point-prevalence studies. First, our study only reflects frequency of toxigenic $C$. difficile detected on 2 days in 2015; therefore, we cannot draw solid conclusions regarding incidence. We expanded the timeframe for assessing the distribution of ribotypes circulating in Switzerland by an additional week for each prevalence day, but this still represents a limited collection of the true incidence. Second, we cannot rule out introduction of bias to testing policies at the participating hospitals, which might have increased testing on the 2 point-prevalence days. However, we did not provide any promotional information regarding our study, so alterations in daily clinical practice among treating physicians is unlikely on these 2 days. Third, we only included liquid stool samples for analyses, but we did not consider any other preanalytical factors, such as the use of laxatives, for testing eligibility. Finally, we applied surveillance definitions recommended by CDC and ECDC rather than defintions used for the clinical diagnosis of CDI in individual patients, such as detection of $C$. difficile in the context of symptoms related to CDI. Therefore, we cannot rule out detection of toxigenic $C$. difficile from colonization rather than infection in some cases.

In conclusion, this nationwide multicenter study reveals that PCR as a stand-alone test results in an increase of $C$. difficile prevalence rates of $\leq 80 \%$ compared with a 2-stage EIA-based algorithm. Our findings underscore the need for consistent testing algorithms for meaningful interinstitutional and nationwide comparisons. Our results also challenge the utility of the current CDC and ECDC case definitions and highlight the need for uniform recommendations on diagnostic approaches.

\section{Acknowledgments}

We acknowledge and thank the ESCMID (European Society of Clinical Microbiology and Infectious Diseases). Study group for C. difficile (ESGCD) for professional support. We also thank all participating centers and laboratories (Appendix, https://wwwnc/cdc.gov/EID/ article/26/10/19-0804-App1.pdf).

Astellas Pharmaceuticals Europe provided financial support for this study. The funder did not influence the study design and did not contribute to data collection, 
data analysis, data interpretation, or writing of the report. Astellas Pharma Europe reviewed the report for factual accuracy before submission, in line with the terms of the funding agreement. The corresponding author had full access to all data in the study and had final responsibility for the decision to submit for publication. Alere provided C. DIFF QUIK CHEK COMPLETE test kits for conducting EIAs to detect GDH and toxins $\mathrm{A}$ and $\mathrm{B}$.

The authors declare the following possible conflicts of interest: A.W. is a member of the Astellas and Merck Sharp \& Dohme Corp. advisory boards for C. difficile and reports grants from the Swiss National Science Foundation. S.T.-S. is a member of the Astellas and Merck Sharp \& Dohme Corp. advisory boards for C. difficile and reports grants from the Swiss National Science Foundation (grant nos. NRP72 and 407240_167060), the Gottfried und Julia Bangerter-Rhyner Stiftung, and the Fund for the Promotion of Teaching and Research of the Voluntary Academic Society, Basel.

\section{About the Author}

Dr. Widmer is head of the infection control program at University Hospital Basel, University of Basel, Switzerland. His research interests include all aspects of Clostridioides difficile and the epidemiology and prevention of hospital-acquired infections.

\section{References}

1. Bartlett JG, Moon N, Chang TW, Taylor N, Onderdonk AB. Role of Clostridium difficile in antibiotic-associated pseudomembranous colitis. Gastroenterology. 1978;75:77882. https:/ / doi.org/10.1016/0016-5085(78)90457-2

2. McDonald LC, Owings M, Jernigan DB. Clostridium difficile infection in patients discharged from US short-stay hospitals, 1996-2003. Emerg Infect Dis. 2006;12:409-15. https://doi.org/10.3201/eid1203.051064

3. Elixhauser A, Jhung M. Clostridium difficile-associated disease in U.S. hospitals, 1993-2005: statistical brief \#50; Healthcare Cost and Utilization Project (HCUP). Rockville (MD, USA): Agency for Healthcare Research and Quality; 2008 [cited 2019 May 26]. https:/ / www.hcup-us.ahrq.gov/reports/ statbriefs/sb50.jsp

4. Jarvis WR, Schlosser J, Jarvis AA, Chinn RY. National point prevalence of Clostridium difficile in US health care facility inpatients, 2008. Am J Infect Control. 2009;37:263-70. https://doi.org/10.1016/j.ajic.2009.01.001

5. Magill SS, Edwards JR, Bamberg W, Beldavs ZG, Dumyati G, Kainer MA, et al.; Emerging Infections Program HealthcareAssociated Infections and Antimicrobial Use Prevalence Survey Team. Multistate point-prevalence survey of health care-associated infections. N Engl J Med. 2014;370:1198-208. https://doi.org/10.1056/NEJMoa1306801

6. Pépin J, Saheb N, Coulombe MA, Alary ME, Corriveau MP, Authier S, et al. Emergence of fluoroquinolones as the predominant risk factor for Clostridium difficile-associated diarrhea: a cohort study during an epidemic in Quebec. Clin Infect Dis. 2005;41:1254-60. https://doi.org/10.1086/496986
7. Loo VG, Poirier L, Miller MA, Oughton M, Libman MD, Michaud S, et al. A predominantly clonal multi-institutional outbreak of Clostridium difficile-associated diarrhea with high morbidity and mortality. N Engl J Med. 2005;353:2442-9. https://doi.org/10.1056/NEJMoa051639

8. O'Donoghue C, Kyne L. Update on Clostridium difficile infection. Curr Opin Gastroenterol. 2011;27:38-47. https://doi.org/10.1097/MOG.0b013e3283411634

9. Lessa FC, Mu Y, Bamberg WM, Beldavs ZG, Dumyati GK, Dunn JR, et al. Burden of Clostridium difficile infection in the United States. N Engl J Med. 2015;372:825-34. https:// doi.org/10.1056/NEJMoa1408913

10. Bauer MP, Notermans DW, van Benthem BH, Brazier JS, Wilcox MH, Rupnik M, et al.; ECDIS Study Group. Clostridium difficile infection in Europe: a hospital-based survey. Lancet. 2011;377:63-73. https://doi.org/10.1016/ S0140-6736(10)61266-4

11. Davies KA, Longshaw CM, Davis GL, Bouza E, Barbut F, Barna Z, et al. Underdiagnosis of Clostridium difficile across Europe: the European, multicentre, prospective, biannual, point-prevalence study of Clostridium difficile infection in hospitalised patients with diarrhoea (EUCLID). Lancet Infect Dis. 2014;14:1208-19. https:/ / doi.org/10.1016/ S1473-3099(14)70991-0

12. US Centers for Disease Control and Prevention. Clostridioides difficile infection (CDI) tracking. [cited 2019 May 26]. https://www.cdc.gov/hai/eip/cdiff-tracking

13. European Centre for Disease Prevention and Control. Surveillance and disease data for Clostridium difficile infections. [cited 2019 May 26]. https:/ / ecdc.europa.eu/ en/clostridium-difficile-infections/surveillance-anddisease-data

14. von Elm E, Altman DG, Egger M, Pocock SJ, Gøtzsche PC, Vandenbroucke JP; STROBE Initiative. The Strengthening the Reporting of Observational Studies in Epidemiology (STROBE) statement: guidelines for reporting observational studies. Lancet. 2007;370:1453-7. https:/ / doi.org/10.1016/ S0140-6736(07)61602-X

15. Stubbs SL, Brazier JS, O'Neill GL, Duerden BI. PCR targeted to the 16S-23S rRNA gene intergenic spacer region of Clostridium difficile and construction of a library consisting of 116 different PCR ribotypes. J Clin Microbiol. 1999; 37:461-3. https://doi.org/10.1128/JCM.37.2.461-463.1999

16. Crobach MJ, Planche T, Eckert C, Barbut F, Terveer EM, Dekkers OM, et al. European Society of Clinical Microbiology and Infectious Diseases: update of the diagnostic guidance document for Clostridium difficile infection. Clin Microbiol Infect. 2016;22(Suppl 4):S63-81. https:// doi.org/10.1016/j.cmi.2016.03.010

17. McDonald LC, Gerding DN, Johnson S, Bakken JS, Carroll KC, Coffin SE, et al. Clinical practice guidelines for Clostridium difficile infection in adults and children: 2017 update by the Infectious Diseases Society of America (IDSA) and Society for Healthcare Epidemiology of America (SHEA). Clin Infect Dis. 2018;66:987-94. https:/ / doi.org/ 10.1093/cid/ciy149

18. Kraft CS, Parrott JS, Cornish NE, Rubinstein ML, Weissfeld AS, McNult P, et al. A laboratory medicine best practices systematic review and meta-analysis of nucleic acid amplification tests (NAATs) and algorithms including NAATs for the diagnosis of Clostridioides (Clostridium) difficile in adults. Clin Microbiol Rev. 2019;32:e0032-18. https:/ / doi.org/10.1128/CMR.00032-18

19. Fenner L, Frei R, Gregory M, Dangel M, Stranden A, Widmer AF. Epidemiology of Clostridium difficile-associated disease at University Hospital Basel including molecular characterisation of the isolates 2006-2007. Eur J Clin 
Microbiol Infect Dis. 2008;27:1201-7. https:/ / doi.org/ 10.1007/s10096-008-0564-9

20. Vernaz N, Sax H, Pittet D, Bonnabry P, Schrenzel J, Harbarth S. Temporal effects of antibiotic use and hand rub consumption on the incidence of MRSA and Clostridium difficile. J Antimicrob Chemother. 2008;62:601-7. https://doi.org/10.1093/jac/dkn199

21. Kohler P, Bregenzer-Witteck A, Rafeiner P, Schlegel M. Presumably hospital-transmitted Clostridium difficile infections based on epidemiological linkage. Swiss Med Wkly. 2013; 143:w13824. https://doi.org/10.4414/smw.2013.13824

22. Goorhuis A, Bakker D, Corver J, Debast SB, Harmanus C, Notermans DW, et al. Emergence of Clostridium difficile infection due to a new hypervirulent strain, polymerase chain reaction ribotype 078. Clin Infect Dis. 2008;47:1162-70. https://doi.org/10.1086/592257
23. Patterson L, Wilcox MH, Fawley WN, Verlander NQ, Geoghegan L, Patel BC, et al. Morbidity and mortality associated with Clostridium difficile ribotype 078: a case-case study. J Hosp Infect. 2012;82:125-8. https://doi.org/10.1016/j.jhin.2012.07.011

24. Romano V, Pasquale V, Krovacek K, Mauri F, Demarta A, Dumontet $\mathrm{S}$. Toxigenic Clostridium difficile PCR ribotypes from wastewater treatment plants in southern Switzerland. Appl Environ Microbiol. 2012;78:6643-6. https:/ / doi.org/10.1128/AEM.01379-12

Address for correspondence: Sarah Tschudin-Sutter, MD, MSc; Division of Infectious Diseases and Hospital Epidemiology, University Hospital Basel, Petersgraben 4, CH-4031 Basel, Switzerland; email: sarah.tschudin@usb.ch

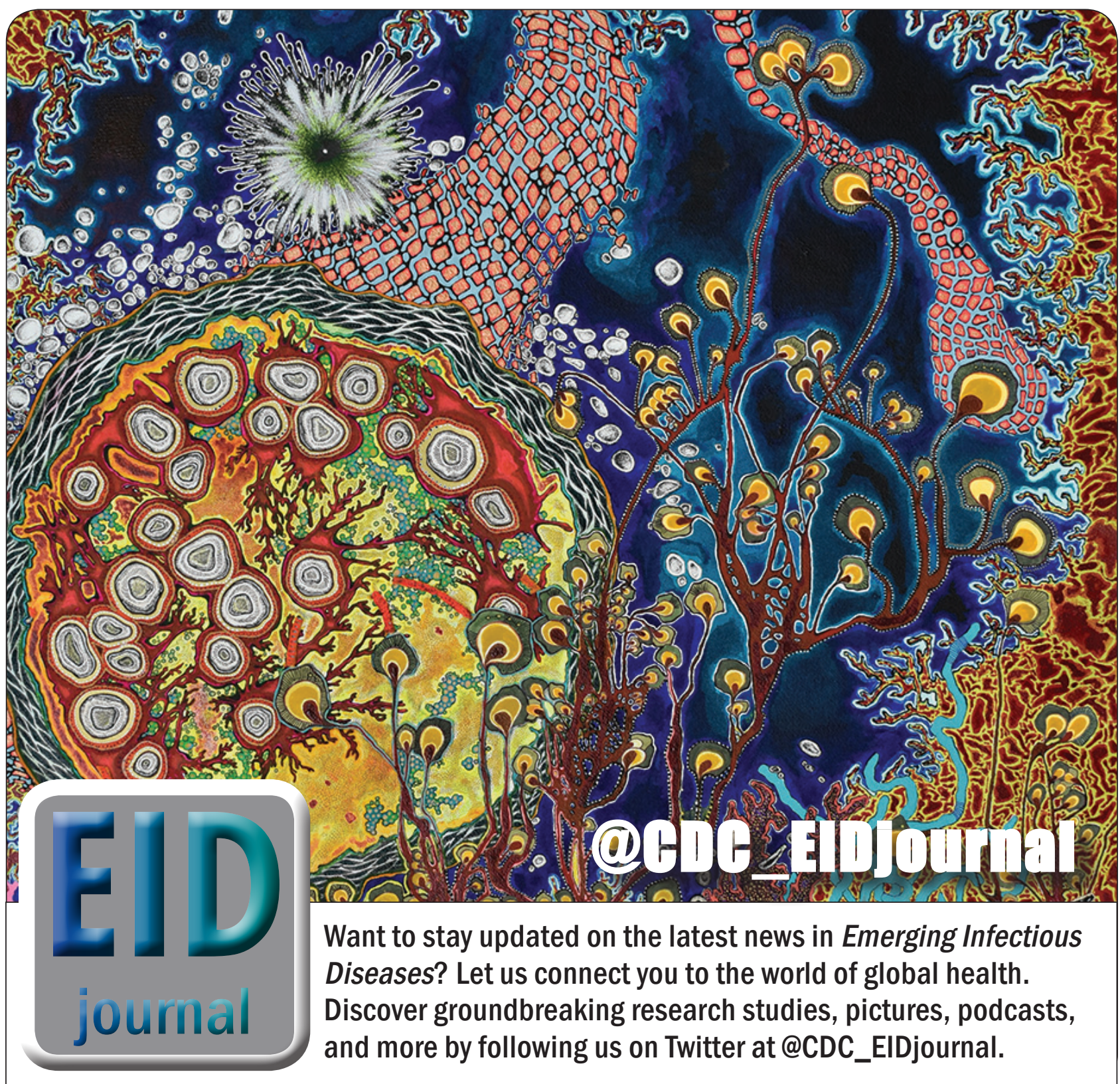

